Published in final edited form as:

Neurology. 2005 April 26; 64(8): 1384-1390.

\title{
The natural history of temporal variant frontotemporal dementia
}

\author{
W.W. Seeley, MD, A.M. Bauer, MD, B.L. Miller, MD, M.L. Gorno-Tempini, MD, PhD, J.H. Kramer, \\ PsyD, M. Weiner, MD, and H.J. Rosen, MD \\ From the Departments of Neurology (Drs. Seeley, Miller, Gorno-Tempni, Kramer, Weiner, and \\ Rosen) and Radiology (Dr. Weiner), USCF Memory and Aging Center (Drs. Seeley, Miller, Gorno- \\ Tempni, Kramer, and Rosen), University of California at San Francisco, San Francisco, CA; \\ Department of Psychiatry (Dr. Bauer), McLean Hospital, San Francisco, CA; Magnetic Resonance \\ Imaging Unit (Dr. Weiner), San Francisco Veterans Affairs Hospital, San Francisco, CA. Supported \\ by the Janssen Foundation (W.W.S.), the John Douglas French Foundation (M.L.G.T.), and a K08 \\ (H.J.R.) and grants from the National Institute of Aging (1P01 AG19724-01A1, P50 \\ AG1657303-75271) and the State of California Department of Health Services
}

\begin{abstract}
Background-The temporal variant of frontotemporal dementia (tvFTD) features asymmetric anterior temporal/amygdala degeneration as well as ventromedial frontal, insular, and inferoposterior temporal involvement. Left temporal atrophy has been linked to loss of semantic knowledge, whereas behavioral symptoms dominate the right temporal variant.
\end{abstract}

Objective-To investigate the first symptoms and the timing of subsequent symptoms in patients with left versus right tvFTD.

Methods-Twenty-six patients with tvFTD were identified. Six had right > left temporal atrophy (right temporal lobe variant [RTLV]) and were matched with six having comparable left > right temporal atrophy (left temporal lobe variant [LTLV]). Clinical records were reviewed to generate individualized symptom chronologies.

Results-In all patients, first symptoms involved semantics (4/6 LTLV, 1/6 RTLV), behavior (4/6 RTLV, 1/6 LTLV), or both (1 LTLV, 1 RTLV). Semantic loss began with anomia, word-finding difficulties, and repetitive speech, whereas the early behavioral syndrome was characterized by emotional distance, irritability, and disruption of physiologic drives (sleep, appetite, libido). After an average of 3 years, patients developed whichever of the two initial syndromes-semantic or behavioral - that they lacked at onset. A third stage, 5 to 7 years from onset, saw the emergence of disinhibition, compulsions, impaired face recognition, altered food preference, and weight gain. Compulsions in LTLV were directed toward visual, nonverbal stimuli, whereas patients with RTLV were drawn to games with words and symbols.

Conclusions-The temporal variant of frontotemporal dementia follows a characteristic cognitive and behavioral progression that suggests early spread from one anterior temporal lobe to the other. Later symptoms implicate ventromedial frontal, insular, and inferoposterior temporal regions, but their precise anatomic correlates await confirmation.

Frontotemporal lobar degeneration (FTLD) describes a group of disorders that includes a frontal variant (fvFTD, or frontotemporal dementia, FTD), a temporal variant (tvFTD, also known as semantic dementia), and nonfluent progressive aphasia. Each disorder bears a signature set of presenting symptoms and regional atrophy patterns. ${ }^{1,2}$ Still understudied,

Address correspondence and reprint requests to Dr. William W. Seeley, Memory and Aging Center, UCSF Department of Neurology, 350 Parnassus Avenue, Suite 706, Box 1207, San Francisco, CA 94143-1207; e-mail: wseeley@ memory.ucsf.edu. 
however, are the stages through which these functional and anatomic deficits evolve. In particular, characterization of the earliest symptoms is needed to facilitate earlier diagnosis.

At presentation, patients with tvFTD show atrophy of the anterior temporal cortex, amygdala, anterior insula, posterior ventromedial frontal cortex,${ }^{1}$ and the temporooccipital gyri. ${ }^{3}$ The anterior temporal atrophy is often asymmetric. ${ }^{4}$ Patients with dominant hemisphere disease lose semantic knowledge, ${ }^{5}$ whereas nondominant degeneration is characterized by prominent emotional and behavioral changes. ${ }^{6,7}$ A recent large study catalogued symptoms in left versus right predominant tvFTD, with groups determined by a radiologist's judgment of the more atrophic side. ${ }^{8}$ Despite considerable symptom overlap, word-finding difficulties and impaired comprehension were more common in the left-sided group, in contrast with person-recognition problems and social behavior changes in the right temporal variant. Initial visits occurred an average of 3.5 years after symptom onset, and patients with left temporal lobe variant (LTLV) outnumbered those with right temporal lobe variant (RTLV) at a rate greater than three to one. Data on the timing of symptoms were not presented.

The gold standard for charting the earliest stages of a neurodegenerative disease remains prospective symptom and imaging analysis, but such an approach is difficult in tvFTD due to its low incidence and the paucity of cases recognized in the preclinical or early clinical phase. In this study, we examined clinical records to estimate first symptoms and the timing of subsequent symptoms in quantitatively defined patients with left- and right-sided tvFTD. Our goal was to chart the natural history of the disease, suspecting that its earliest symptoms would involve semantics in LTLV and behavior in RTLV, but that the two syndromes would converge before presentation.

\section{Methods}

\section{Subjects}

Patients-Fifty-eight patients with a research diagnosis of FTD or semantic dementia ${ }^{9}$ were identified from the UCSF Memory and Aging Center (MAC) database. All had undergone an in-depth evaluation by a neurologist (B.L.M), a nurse, and a neuropsychologist (J.H.K). The neuropsychological screening battery, described in detail elsewhere, 10 was designed to quickly and reliably assess episodic memory, working memory, executive functioning, fluency, naming, semantics, syntax, spatial ability, and abstract reasoning. The Neuropsychiatric Inventory (NPI) ${ }^{11}$ quantified patients' behavioral symptoms in a cross-sectional manner at presentation.

Controls-Twenty imaging control subjects (10 men, 10 women, mean age $66.8 \pm 8.4$ years) were chosen to match the patient groups in age. None had a history of neurologic or psychiatric disorders or an MRI scan with evidence of focal disease or subcortical white matter ischemia. Because neuropsychological data were not available on all imaging controls, 12 different healthy subjects were chosen to match the patients in age and serve as cognitive controls.

\section{Acquisition of MRI and cerebral volumes}

MRI and tissue segmentation-Structural MRI was accomplished using a 1.5-T Magnetom VISION system (Siemens Inc., Iselin, NJ), a standard quadrature head coil, and previously described sequences and protocols $1,12,13$ to segment the entire brain into gray matter, white matter, and CSF.

Cerebral volume measurements-Regions of interest (ROIs) were manually traced, yielding volumes for the left and right anterior temporal cortex (ATC), amygdala, ventromedial frontal cortex (VMFC), and total frontal cortex (TFC), as previously described. ${ }^{10,12}$ Regions 
selected had shown volume loss in tvFTD in previous whole-brain analyses performed in our laboratory. ${ }^{1}$ ROI volumes for each patient were compared with corresponding regions in the imaging control group, and a $Z$ score was created for each region in each patient.

Patient classification-For the 58 clinically selected patients with FTLD, bilateral temporal and total frontal $Z$ scores were used to sort patients into temporal and frontal variants, depending on which region had the more negative mean $Z$ score, provided that the $Z$ score for the frontal and temporal regions in that patient differed by at least 0.5 . Twenty-five patients met this criterion for tvFTD and were then designated as LTLV or RTLV based on which temporal lobe had the more negative mean $Z$ score. Only six patients were found to have greater right temporal atrophy (RTLV), consistent with prior studies suggesting that LTLV is roughly three times more prevalent. 8 The patients with RTLV varied with respect to temporal atrophy severity and asymmetry. Accordingly, from the remaining 19 patients with LTLV, six were chosen to mirror the RTLV group as closely as possible (figure 1). Comparable degrees of temporal atrophy severity (averaged temporal volume $Z$ score: RTLV mean -3.29 , range -2.67 to -3.58 ; LTLV mean -3.27 , range -2.36 to -4.15 ) and asymmetry (temporal volume $Z$ score difference: RTLV mean 0.90 , range 0.34 to 1.59 ; LTLV mean 0.82 , range 0.13 to 1.40 ) were achieved. Of note, though slightly less homogeneous, the 13 patients with LTLV not included in the analysis showed temporal atrophy severity (averaged temporal volume $Z$ score: mean -3.32 , range -0.76 to -6.59 ) and asymmetry (temporal volume $Z$ score difference: mean 1.31 , range 0.13 to 3.03 ) not statistically different from the patients with tvFTD analyzed. Among the final 12 patients described, all were right handed but one (RTLV 1).

\section{Symptom ascertainment}

First symptoms were routinely recorded during neurologic interviews at our center, prompted by use of a semistructured interview form designed to elicit a broad range of dementia symptoms, including those characteristic of atypical dementias. Clinical records for the 12 patients with tvFTD, including notes from outside physicians, were examined by one reviewer (A.M.B.) who could not be blinded to patient classification. To reduce abstraction bias and to explore all potential early manifestations of tvFTD, the reviewer abstracted every symptom present in the records to produce an individualized symptom chronology for each patient. For each symptom, an interval from disease onset was estimated and rounded to the nearest year. First symptoms were assigned an interval of zero. Individual first symptoms, all of which could be classified as semantic or behavioral, were grouped to create first symptom category "clusters," so that the time from disease onset to development of the "opposite first symptom cluster" (a behavioral first symptom cluster symptom if a patient's first symptoms were semantic and vice versa) could be assessed.

\section{Compulsion types}

Given the prevalence of compulsions in our patients, we chose to examine the environmental stimuli toward which compulsions were driven. Patients were classified as targeting stimuli that were 1) visual, if they were objects devoid of verbal information whose meaning is derived through appreciation of form (e.g., sticks, coins, pictures); 2) verbal/symbolic, if they were composed primarily of letters, words, or symbols (e.g., playing cards); or 3) both, if both visual and verbal/symbolic compulsions were pursued by that patient.

\section{Statistical analyses}

Patient characteristics and neuropsychological performance-Group effects on demographic and neuropsychological variables were assessed using one-way analyses of variance. Post hoc pairwise group comparisons were made using the Tukey procedure, correcting for multiple comparisons. 
Regional brain volumes-The regional volume analysis had three primary objectives. First, we sought to determine the degree to which measured brain regions were reduced in volume relative to each other. For this purpose, ROIs were designated as ipsilateral or contralateral, using the side of worst temporal atrophy as the point of reference. $Z$ scores were then subjected to paired-sample $t$ tests using a within-subjects design that combined patients with LTLV and patients with RTLV into a single tvFTD group. Second, we hoped to address the question of whether LTLV and RTLV develop comparable atrophy in extratemporal ROIs when matched for temporal involvement. Thus, between-group differences in ipsilateral and contralateral ROI $Z$ scores were assessed using independent-sample $t$ tests. Finally, we used one-tailed Pearson correlations to test the hypothesis that disease duration would correlate negatively with volumes in individual ROIs.

Symptom timing-Because 61 distinct tvFTD symptoms were identified, interval data from two subsets were chosen for further scrutiny: 1) symptoms that were part of the semantic or behavioral first symptom cluster and 2) any symptom, not included in 1) that occurred in at least nine of the 12 patients. Patients in whom a symptom of interest was not present in the record were excluded from the analysis for that symptom only. To examine whether the opposite first symptom cluster came earlier than the other common downstream symptoms (subset 2 above), we compared mean intervals from disease onset using paired-sample $t$ tests.

Compulsion types-Chi-square analysis was used to examine group effects on the compulsion target category. All statistical analyses were accomplished using the SPSS software package (version 10.0.5 for Windows, SPSS Inc., Chicago, IL).

\section{Results}

\section{Group characteristics and neuropsychological data}

Demographic variables and neuropsychological profiles at presentation are compared in table 1. Mean duration of symptoms before our evaluation did not differ between groups (LTLV 7.4 years, $\mathrm{SD}=3.9$, range 3 to 12.5 ; RTLV 6.6 years, $\mathrm{SD}=2.9$, range 2.5 to 10.5 ). Sentence comprehension was reduced in LTLV but not RTLV when compared with controls. Both groups were impaired on the 15-item Boston Naming Test, but LTLV showed a significantly greater deficit. In contrast, although the RTLV group was not demonstrably worse than controls on the figure-copying task, their performance was significantly below that of the patients with LTLV. No differences in NPI ratings reached statistical significance.

\section{Regional brain volumes}

Mean volume $Z$ scores for manually traced ROIs are shown in figure 2 . In keeping with the study design, LTLV and RTLV groups were well matched for the degrees of temporal atrophy on the ipsilateral ( $L T L V Z=-3.68, \mathrm{SD}=0.76$; $\mathrm{RTLV} Z=-3.74, \mathrm{SD}=0.45$ ) and contralateral ( $L T L V Z=-2.86, \mathrm{SD}=0.69 ; \mathrm{RTLV} Z=-2.83, \mathrm{SD}=0.37$ ) sides. As expected, in both groups, the ipsilateral temporal lobe was the most atrophied, statistically more so than the next most atrophied regions, the contralateral temporal lobe (LTLV: $t=4.64, p=0.006$; RTLV: $t=5.08$; $p=0.004$ ) and ipsilateral amygdala (LTLV: $t=4.08$; RTLV: $t=4.07$; both $p=0.010$ ). The contralateral temporal lobe, in turn, was more atrophied than ipsilateral VMFC (LTLV: $t=$ $2.75, p=0.04$; RTLV: $t=3.76, p=0.01$ ), contralateral VMFC (LTLV: $\mathrm{t}=4.72, p=0.005$ RTLV: $\mathrm{t}=3.85, p=0.01$ ), and contralateral TFC (LTLV: $t=4.64, p=0.006$; RTLV: $t=4.55$, $p=0.006)$. In RTLV, contralateral temporal atrophy was also greater than in the ipsilateral TFC (RTLV: $t=3.70, p=0.01$ ), but this trend did not reach significance in the LTLV group (LTLV: $t=2.17, p=0.08$ ). When all 12 patients were examined together, the significant findings seen at the subgroup level were again demonstrated (figure 2). Between groups, the 
only differing region was the ipsilateral amygdala, in which RTLV showed more atrophy $(t=$ $4.65, p=0.001)$ than LTLV.

Analyzing all 12 patients together, anterior temporal volumes strongly and inversely correlated with disease duration (total: $r=-0.71, p=0.005$; ipsilateral: $r=-0.67, p=0.009$; contralateral: $r=-0.66, p=0.01$ ). Ipsilateral VMFC volume showed a trend in the same direction (Pearson $r=-0.47, p=0.06$ ). No other ROI was significantly related to disease duration. Because ipsilateral amygdala volume differed between groups (RTLV < LTLV), we examined its relationship to disease duration separately for the two variants. In RTLV, there was a trend toward longer symptom duration predicting greater ipsilateral amygdala volume loss $(r=$ $-0.65, p=0.08)$, whereas in LTLV this relationship was not identified $(r=-0.28, p=0.30)$.

\section{First symptoms}

The initial manifestations of tvFTD are shown in table 2, which highlights the first symptoms of the illness (marked with asterisks) as well as the first symptoms to evolve in semantics and behavior for each patient. TVFTD began with semantics alone in four of six patients with LTLV and one of six patients with RTLV. In contrast, an isolated behavioral disturbance marked disease onset in four of six patients with RTLV and one of six patients with LTLV. Two patients (one LTLV, one RTLV) had initial symptoms involving both semantics and behavior. Notably, these two patients were the patient with RTLV with the least temporal volume asymmetry (RTLV 5: RATC $Z=-2.84$, LATC $Z=-2.50$ ) and the LTLV patient chosen to match her (LTLV 2: LATC $Z=-2.60$, RATC $Z=-2.47$ ). Independent of group, when semantic loss came first, it manifested as word-finding and naming problems, reduced word comprehension, or repetitive speech. Behavioral symptoms at disease onset formed a complex of depression, irritability/hostility, emotional blunting/withdrawal, apathy, insomnia, poor appetite, weight loss, decreased sexual interest, and increased alcohol intake. These initial symptoms defined the semantic and behavioral first symptom clusters.

\section{Subsequent symptoms}

Figure 3 demonstrates the mean intervals from disease onset to downstream symptoms of interest. The interval to the opposite first symptom cluster was similar regardless of whether the disease began with semantics or behavior. Among the five patients whose disease began with semantic loss alone, the behavioral cluster arose after a mean interval of 3.8 years $(\mathrm{SD}=$ 2.6). Conversely, the five with an initial behavioral syndrome developed semantic loss after 3.2 years $(\mathrm{SD}=2.8)$. The two patients with simultaneous onset lowered the mean intervals to opposite first symptom cluster shown in figure 3. Despite the multifold behavioral symptoms of tvFTD, only two patients with semantic loss at disease onset (LTLV 1 and 4) had a first behavioral symptom outside the behavioral first symptom cluster.

Disinhibition (six LTLV, five RTLV), compulsions (five LTLV, six RTLV), face recognition problems (three LTLV, six RTLV), altered food preference (six LTLV, three RTLV), and weight gain (five LTLV, five RTLV) were the only other symptoms to occur in at least nine of 12 patients. Despite their high prevalence, none of these symptoms ever occurred as a first symptom. Neither prevalence nor time to onset of these later symptoms differed statistically between groups. However, examining all patients with tvFTD together, the opposite first symptom cluster (behavioral symptoms if the disease began with semantics and vice versa) arose earlier than the other most common downstream symptoms (opposite first symptom cluster vs disinhibition: $t=3.89, d f=10, p=0.003$; vs compulsions: $t=2.31, d f=10, p=0.04$; vs face recognition: $t=4.62, d f=8, p=0.002$; vs altered food preference: $t=4.10, d f=8, p=$ 0.003 ; vs weight gain: $t=3.39, d f=9, p=0.008$ ). Changes in food preference, when specified, included eating frozen or rotten food, avoiding specific food classes (protein, spicy foods), seeking lipid- and carbohydrate-rich food items (sweets, fast food, potato chips), and restrictive 
dieting (eating only chicken, rice, and bananas). Other symptoms occurring in at least six of 12 patients but never as first symptoms included loss of empathy (three LTLV, four RTLV) and mental rigidity (four LTLV, two RTLV).

\section{Compulsive behaviors}

Compulsions were a prominent but often delayed symptom, occurring in 11 of 12 patients. As shown in table 3, the target stimuli of LTLV compulsions were more often objects, freely available in the environment, with salience of a visual nature (visual/nonverbal, three; verbal/ symbolic, none; both, two). In contrast, patients with RTLV were focused on letters, words, and symbols (visual/nonverbal, none; verbal/symbolic, four; both, two). The interaction between group and stimulus type was significant (Pearson $\chi^{2}=6.97, p<0.05$ ).

\section{Discussion}

In this study, the first to examine the timing of symptoms in the tvFTD, we reviewed the clinical records of six patients with left and six patients with right tvFTD matched for severity and asymmetry of anterior temporal involvement. Our findings suggest that the earliest symptoms of tvFTD depend on the side of predominant temporal degeneration. When the disease begins with semantic loss, the left temporal lobe is more markedly affected. In contrast, behavioral changes at onset herald right temporal degeneration. Despite these initial differences, the two variants progressively merge. After an average of 3 years, the second symptom type to arise is most often the one, semantic or behavioral, not present at inception. After 5 to 7 years, disinhibition, compulsions, face recognition problems, altered food preference, and weight gain emerge. A first evaluation is typically sought during this stage of the illness, perhaps due to rising caregiver distress. The longer symptoms go on before evaluation, the more severe the bitemporal atrophy is at initial imaging.

In our sample, patients with tvFTD were symptomatic an average of 7 years before presentation. In an uncommon dementia with such an insidious disease course, it is difficult to collect prospective natural history data. The retrospective method used here provides a viable alternative but brings with it potential biases relating to informant recall and symptom abstraction. Informants may have reported the most striking, rather than the earliest, changes when queried about first symptoms. We attempted to reduce abstraction bias by pulling out each symptom for each patient, while focusing on first and most common symptoms in the subsequent analyses. Although our sample was small, its cases were defined by matching temporal atrophy severity and asymmetry scores that were similar to those of the 13 patients with LTLV not included in the study, suggesting that our natural history data may generalize to other patients with tvFTD.

The anterior temporal lobes remain largely uncharted brain regions, in part due to the scarcity of naturally occurring isolated temporopolar lesions. Elegant studies of semantic dementia (LTLV) have begun to reveal how the left anterior temporal lobe supports semantic knowledge, 14 but less is known about anterior temporal contributions to social and emotional behavior. Anterior temporal lesions impair emotional attachment to family, as reported in an adult after right temporal lobectomy 15 and children recovering from herpes encephalitis. ${ }^{16}$ Deficits reading facial affect are seen in early right mesiotemporal epilepsy ${ }^{17}$ and after right temporal lobectomy 18 and correlate with right amygdala and ventromedial frontal cortex atrophy in tvFTD. ${ }^{12}$ In the present study, related behavioral functions were disrupted early in the course of tvFTD. Right temporoamygdalar degeneration began with emotional distance (blunting, withdrawal, apathy, depression), irritability, and altered physiologic drives (sleep, appetite, libido). LTLV typically began with semantic loss, but its first behavioral symptoms largely simulated those seen at RTLV onset. Further, the 3-year semantic prelude to the behavioral first symptom cluster in LTLV was echoed in RTLV, which averaged 3 years of behavioral 
change before semantic loss took hold. Given the symmetry of these profiles and the close link between disease duration and anterior temporal atrophy, we propose that tvFTD becomes bitemporal early in its course.

If the early symptoms of tvFTD reflect its anterior temporal origins, what might its later evolving symptoms tell us about its anatomic progression? In this cohort, the most common downstream symptoms began an average of 5 to 7 years into the illness. Disinhibition and compulsions, the most ubiquitous later symptoms, have been associated with ventromedial and orbital frontal cortex dysfunction. ${ }^{19,20}$ Problems with face recognition are known to occur in $\mathrm{RTLV}^{8,21,22}$ and likely reflect progressive inferior temporal cortex injury, especially on the right. ${ }^{23}$ Altered food preferences suggest misprocessing of insular disgust signals ${ }^{24}$ in some patients and derailed satiety controls, perhaps due to orbital frontal pathology, in others. ${ }^{25}$ Weight gain may result from appetite dysregulation, the hyperorality of bilateral temporoamygdalar damage, ${ }^{26}$ the motor inactivity of dorsomedial frontal involvement, ${ }^{19}$ or a combination. Somehow, patients at this stage of the illness are no longer able to match caloric expenditure to their often copious, idiosyncratic intake. These functional-anatomic

correlations, combined with the natural history data from our patients, should serve as a guide for researchers seeking to establish the regional progression of tvFTD.

Compulsions are a prominent feature in FTLD, ${ }^{27,28}$ trouble family members, and at times respond to treatment, but their neuroanatomic and mechanistic underpinnings are unclear. Some authors emphasize the importance of frontal pathology, ${ }^{29}$ while others stress temporal involvement, ${ }^{30}$ especially when it is asymmetric. ${ }^{4}$ The relevance of striatal pathology is suspected, but attempts to confirm it have failed. ${ }^{4,30}$ Patients with tvFTD are often fixated on their compulsions, tolerate interruption poorly, and adopt rigid performance schedules, leading to the suggestion that tvFTD compulsions are more compulsive than those of fvFTD. ${ }^{30}$ Addressing these issues was not an initial goal of this study, but our data suggest that compulsions occur later in the course of tvFTD than would be expected if asymmetric temporal atrophy were the primary cause. In addition, our data suggest a driving force behind the specific types, and perhaps also the immediacy, of tvFTD compulsions. In LTLV, words lose meaning but visual attention is heightened, and compulsions are focused on visually conspicuous objects. In RTLV, however, complex social and visual esthetic cues are devalued, and patients favor games with letters, words, and playing cards, perhaps to draw meaning from uniform, easily interpretable symbols. Therefore, it appears that temporal lobe asymmetry may perturb environmental stimulus appraisal such that excess salience is conferred on particular stimulus classes. In return, compulsions targeting overvalued stimuli can become a dominant expression of behavior. Further investigation of this preliminary hypothesis is required.

Available imaging data 1,3 combined with the regional volumes presented here suggest that, by presentation, tvFTD has often devastated one temporal lobe, encroached on the other, spread to the amygdala (ipsilateral worse than contralateral, especially in RTLV), and, to a lesser extent, involved the frontal, insular, and inferoposterior temporal cortices. Considering the known functions of these structures, our natural history data provide clinical evidence that regional degeneration in tvFTD occurs in a predictable sequence. Although LTLV appears to be threefold more common than $\mathrm{RTLV}^{8}$ the overlap shown here supports the view that both variants reflect the same pathophysiologic process and that greater left temporal vulnerability to that process should be explored.

\section{Acknowledgements}

The authors thank Megan Bradley at the San Francisco Veterans Affairs Magnetic Resonance Imaging Unit for her help with image acquisition. 


\section{References}

1. Rosen HJ, Gorno-Tempini ML, Goldman WP, et al. Patterns of brain atrophy in frontotemporal dementia and semantic dementia. Neurology 2002;58:198-208. [PubMed: 11805245]

2. Gorno-Tempini ML, Dronkers NF, Rankin KP, et al. Cognition and anatomy in three variants of primary progressive aphasia. Ann Neurol 2004;55:335-346. [PubMed: 14991811]

3. Studholme C, Cardenas V, Blumenfeld R, et al. Deformation tensor morphometry of semantic dementia with quantitative validation. Neuroimage 2004;21:1387-1398. [PubMed: 15050564]

4. Rosso SM, Roks G, Stevens M, et al. Complex compulsive behaviour in the temporal variant of frontotemporal dementia. J Neurol 2001;248:965-970. [PubMed: 11757960]

5. Hodges JR, Patterson K, Oxbury S, Funnell E. Semantic dementia. Progressive fluent aphasia with temporal lobe atrophy. Brain 1992;115:1783-1806. [PubMed: 1486461]

6. Perry RJ, Rosen HR, Kramer JH, Beer JS, Levenson RL, Miller BL. Hemispheric dominance for emotions, empathy and social behaviour: evidence from right and left handers with frontotemporal dementia. Neurocase 2001;7:145-160. [PubMed: 11320162]

7. Edwards-Lee T, Miller BL, Benson DF, et al. The temporal variant of frontotemporal dementia. Brain 1997;120:1027-1040. [PubMed: 9217686]

8. Thompson SA, Patterson K, Hodges JR. Left/right asymmetry of atrophy in semantic dementia: behavioral-cognitive implications. Neurology 2003;61:1196-1203. [PubMed: 14610120]

9. Neary D, Snowden JS, Gustafson L, et al. Frontotemporal lobar degeneration: a consensus on clinical diagnostic criteria. Neurology 1998;51:1546-1554. [PubMed: 9855500]

10. Liu W, Miller BL, Kramer JH, et al. Behavioral disorders in the frontal and temporal variants of frontotemporal dementia. Neurology 2004;62:742-748. [PubMed: 15007124]

11. Cummings JL. The Neuropsychiatric Inventory: assessing psychopathology in dementia patients. Neurology 1997;48(5 suppl 6):S10-16. [PubMed: 9153155]

12. Rosen HJ, Perry RJ, Murphy J, et al. Emotion comprehension in the temporal variant of frontotemporal dementia. Brain 2002;125:2286-2295. [PubMed: 12244085]

13. Tanabe JL, Amend D, Schuff N, et al. Tissue segmentation of the brain in Alzheimer disease. AJNR Am J Neuroradiol 1997;18:115-123. [PubMed: 9010529]

14. Mummery CJ, Patterson K, Price CJ, Ashburner J, Frackowiak RS, Hodges JR. A voxel-based morphometry study of semantic dementia: relationship between temporal lobe atrophy and semantic memory. Ann Neurol 2000;47:36-45. [PubMed: 10632099]

15. Lipson SE, Sacks O, Devinsky O. Selective emotional detachment from family after right temporal lobectomy. Epilepsy Behav 2003;4:340-342. [PubMed: 12791338]

16. Pradhan S, Singh MN, Pandey N. Kluver Bucy syndrome in young children. Clin Neurol Neurosurg 1998;100:254-258. [PubMed: 9879850]

17. Meletti S, Benuzzi F, Rubboli G, et al. Impaired facial emotion recognition in early-onset right mesial temporal lobe epilepsy. Neurology 2003;60:426-431. [PubMed: 12578923]

18. Anderson AK, Spencer DD, Fulbright RK, Phelps EA. Contribution of the anteromedial temporal lobes to the evaluation of facial emotion. Neuropsychology 2000;14:526-536. [PubMed: 11055255]

19. Cummings JL. Anatomic and behavioral aspects of frontal-subcortical circuits. Ann NY Acad Sci 1995;769:1-13. [PubMed: 8595019]

20. Volle E, Beato R, Levy R, Dubois B. Forced collectionism after orbito-frontal damage. Neurology 2002;58:488-490. [PubMed: 11839860]

21. Gainotti G, Barbier A, Marra C. Slowly progressive defect in recognition of familiar people in a patient with right anterior temporal atrophy. Brain 2003;126(pt 4):792-803. [PubMed: 12615639]

22. Snowden JS, Thompson JC, Neary D. Knowledge of famous faces and names in semantic dementia. Brain 2004;127(pt 4):860-872. [PubMed: 14985259]

23. Gorno-Tempini ML, Price CJ, Josephs O, et al. The neural systems sustaining face and proper-name processing. Brain 1998;121(pt 11):2103-2118. [PubMed: 9827770]

24. Wicker B, Keysers C, Plailly J, Royet JP, Gallese V, Rizzolatti G. Both of us disgusted in My insula: the common neural basis of seeing and feeling disgust. Neuron 2003;40:655-664. [PubMed: 14642287] 
25. Rolls ET. Taste and olfactory processing in the brain and its relation to the control of eating. Crit Rev Neurobiol 1997;11:263-287. [PubMed: 9336714]

26. Mendez M, Foti D. Lethal hyperoral behaviour from the Kluver-Bucy. J Neurol Neurosurg Psychiatry 1997;62:293-294. [PubMed: 9069496]

27. Miller BL, Darby AL, Swartz JR, Yener GG, Mena I. Dietary changes, compulsions and sexual behavior in frontotemporal degeneration. Dementia 1995;6:195-199. [PubMed: 7550598]

28. Ames D, Cummings JL, Wirshing WC, Quinn B, Mahler M. Repetitive and compulsive behavior in frontal lobe degenerations. J Neuropsychiatry Clin Neurosci 1994;6:100-113. [PubMed: 8044031]

29. Nyatsanza S, Shetty T, Gregory C, Lough S, Dawson K, Hodges JR. A study of stereotypic behaviours in Alzheimer's disease and frontal and temporal variant frontotemporal dementia. J Neurol Neurosurg Psychiatry 2003;74:1398-1402. [PubMed: 14570833]

30. Snowden JS, Bathgate D, Varma A, Blackshaw A, Gibbons ZC, Neary D. Distinct behavioural profiles in frontotemporal dementia and semantic dementia. J Neurol Neurosurg Psychiatry 2001;70:323332. [PubMed: 11181853] 

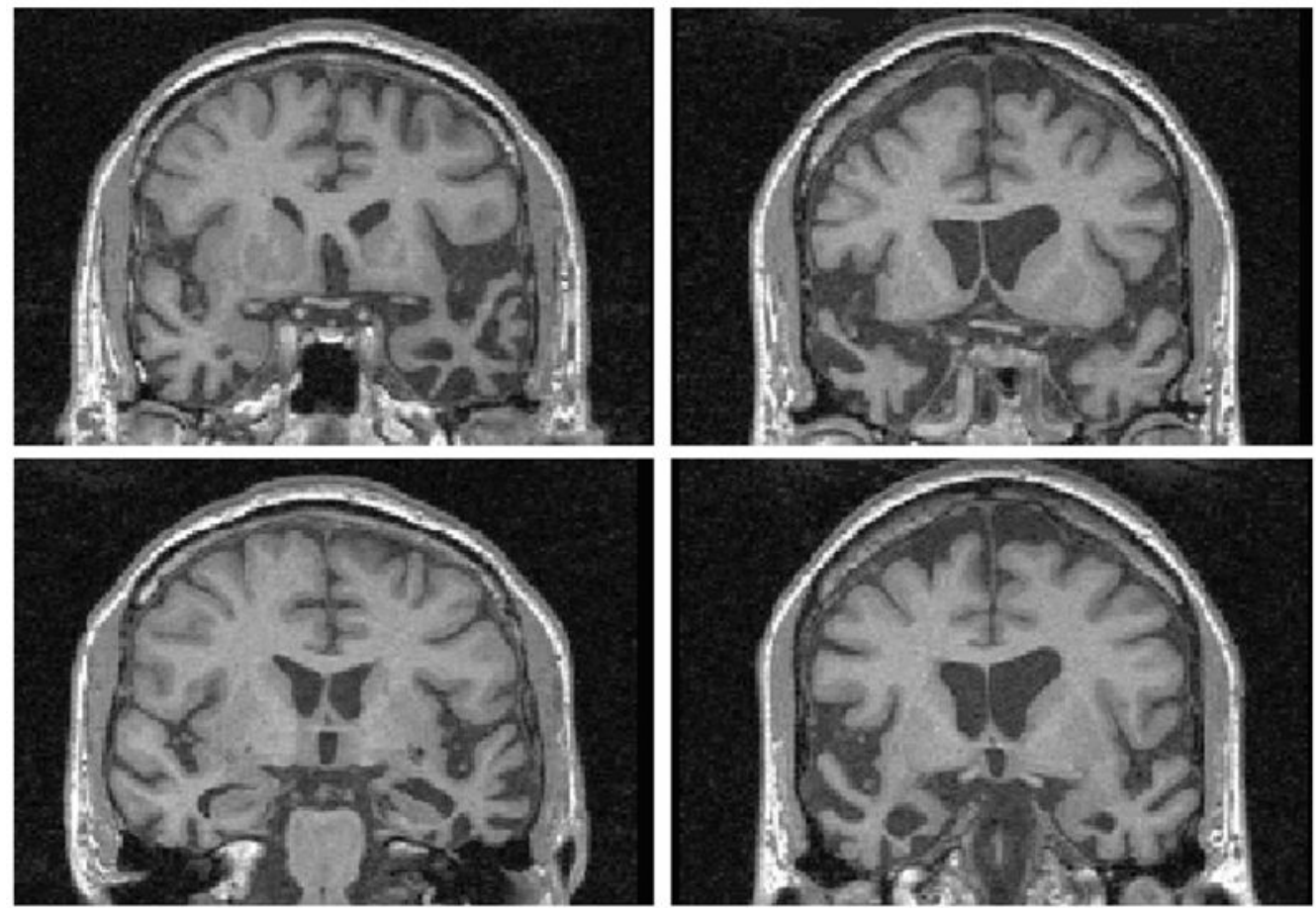

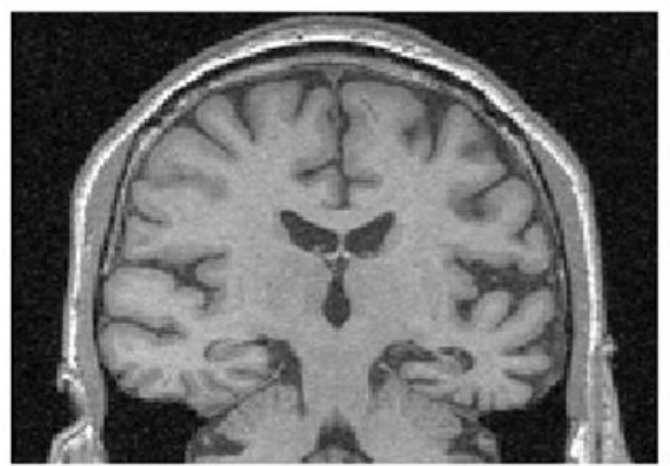

LTLV 1

LATC -3.92

RATC -3.13

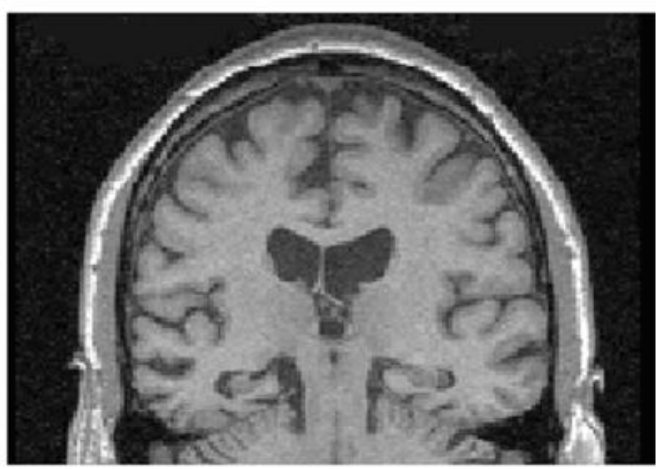

RTLV 6

LATC -3.12

RATC -3.82

Figure 1.

Coronal T1 MP-RAGE images of a representative pairing of patients with left and right temporal lobe variants (LTLV and RTLV) using temporal volume Z scores. The left side of each image represents the right side of the head. LATC $=$ left anterior temporal cortex; RATC $=$ right anterior temporal cortex. 


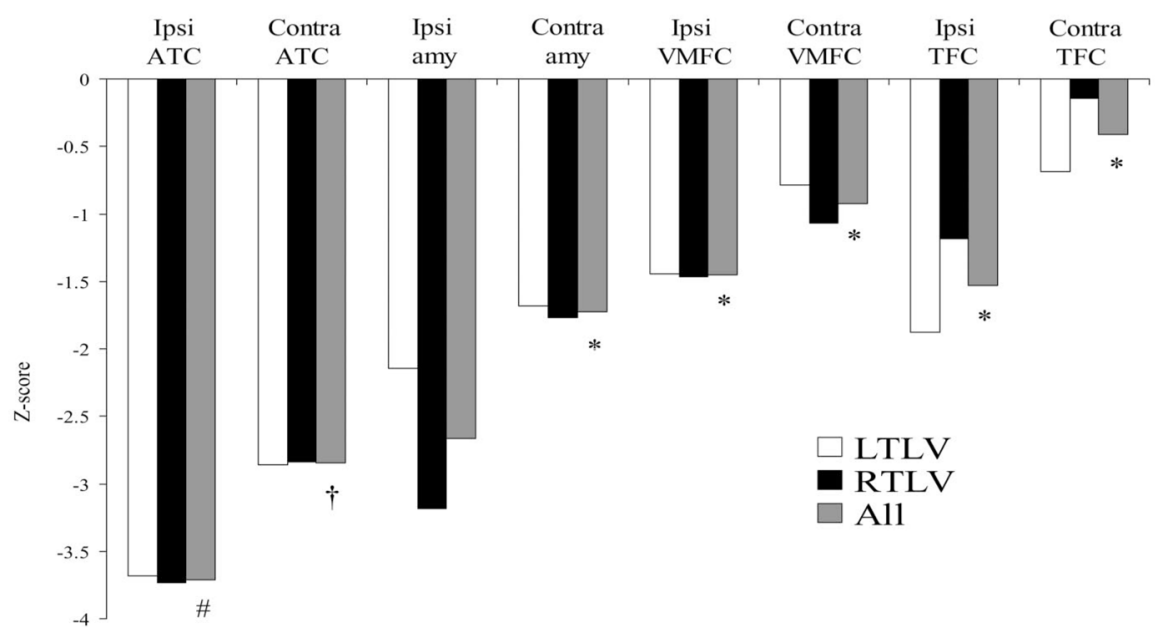

Figure 2.

Mean volume $\mathrm{Z}$ scores for anatomic regions of interest. Reference to side is relative to side of worst temporal atrophy. Ipsi $=$ ipsilateral; Contra $=$ contralateral; ATC $=$ anterior temporal cortex; amy = amygdala; $\mathrm{VMFC}=$ ventromedial frontal cortex; $\mathrm{TFC}=$ total frontal cortex. Significant differences vs mean contralateral temporal $Z$ score $(\dagger)$ indicated by $* p \leq$ $0.006,{ }^{\#} p<0.001$. 


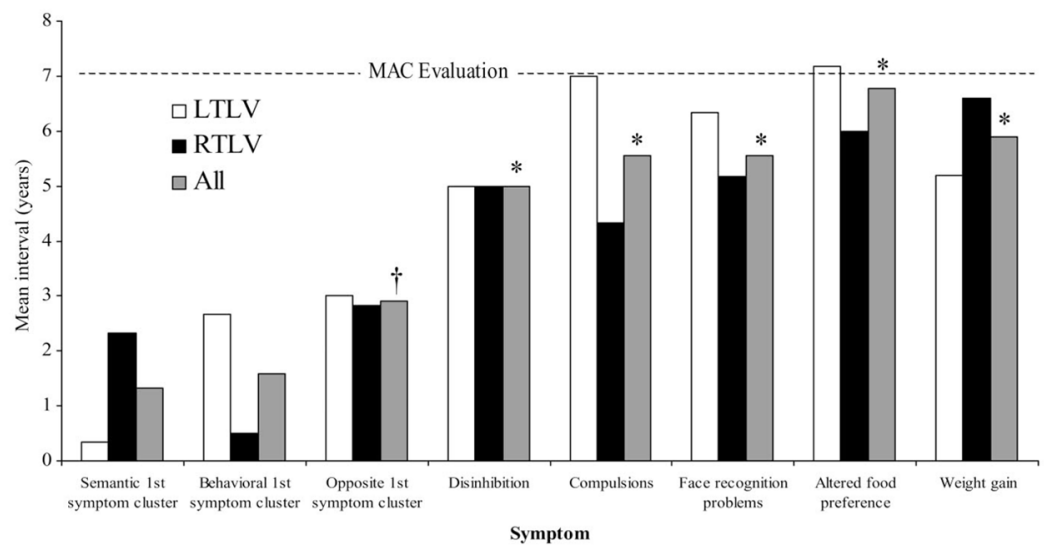

Figure 3.

Intervals to first symptom clusters and downstream symptoms occurring in $\geq 75 \%$ of patients. Symptoms present at onset were assigned a value of zero. Dotted line indicates overall mean duration of symptoms at time of entry. *Mean interval significantly greater than mean interval to opposite first symptom cluster $(\dagger), p<0.05$. LTLV = left temporal lobe variant; RTLV = right temporal lobe variant. 
Table 1

Demographics and neuropsychological test results for normal controls, LTLV, and RTLV

\begin{tabular}{|c|c|c|c|c|}
\hline & Overall ANOVA & $\begin{array}{c}\text { Controls, } \mathbf{n}= \\
12\end{array}$ & LTLV, $n=6$ & RTLV, $n=6$ \\
\hline Age & $\mathrm{F}(2,21)=1.4$ & $61.1(5.5)$ & $64.0(7.8)$ & $66.5(7.6)$ \\
\hline $\mathrm{M} / \mathrm{F}$ & & $5 / 7$ & $2 / 4$ & $4 / 2$ \\
\hline Education & $\mathrm{F}(2,21)=1.9$ & $17.8(2.4)$ & $15.8(2.0)$ & $15.8(2.7)$ \\
\hline Disease duration & $\mathrm{F}(1,10)=0.2$ & N/A & $7.4(3.9)$ & $6.6(2.9)$ \\
\hline MMSE $(\max =30)$ & $\mathrm{F}(2,21)=10.7^{*}$ & $29.8(0.4)$ & $21.2(4.5)^{\dagger}$ & $23.0(7.2)^{\dagger}$ \\
\hline CVLT-MS 30-s free recall $(\max =9)$ & $\mathrm{F}(2,21)=43.8^{*}$ & $8.7(0.6)$ & $1.2(1.3)^{\dagger}$ & $2.5(3.0)^{\dagger}$ \\
\hline CVLT-MS 10 -min free recall $(\max =9)$ & $\mathrm{F}(2,21)=44.0 *$ & $8.4(0.9)$ & $0.6(0.9)^{\dagger}$ & $1.8(3.1)^{\dagger}$ \\
\hline $\begin{array}{l}\text { Modified Rey-Osterrieth Delay }(\max = \\
\text { 17) }\end{array}$ & $\mathrm{F}(2,21)=8.8^{*}$ & $12.5(2.8)$ & $7.5(4.3)^{\dagger}$ & $5.3(4.5)^{\dagger}$ \\
\hline Digit span backward & $\mathrm{F}(2,20)=0.7$ & $5.2(0.8)$ & $4.8(0.8)$ & $4.7(1.2)$ \\
\hline Modified trails no. of lines/min & $\mathrm{F}(2,19)=6.5^{*}$ & $37.1(15.0)$ & $11.0(4.7)^{\dagger}$ & $20.2(18.8)$ \\
\hline Stroop no. correct/min & $\mathrm{F}(2,18)=10.7^{*}$ & $52.8(20.0)$ & $14.2(10.2)^{\dagger}$ & $29.0(13.5)$ \\
\hline Design fluency (novel designs/min) & $\mathrm{F}(2,21)=6.1^{*}$ & $11.0(4.0)$ & $5.3(1.0)^{\dagger}$ & $6.2(4.6)^{t}$ \\
\hline Phonemic fluency (D words/min) & $\mathrm{F}(2,20)=13.3^{*}$ & $14.3(4.3)$ & $6.0(2.4)^{\dagger}$ & $6.8(3.0)^{\dagger}$ \\
\hline Semantic fluency (animals/min) & $\mathrm{F}(2,20)=47.5^{*}$ & $22.6(4.5)$ & $5.2(1.7)^{\dagger}$ & $4.8(5.6)^{\dagger}$ \\
\hline Abbreviated BNT $(\max =15)$ & $\mathrm{F}(2,20)=65.1^{*}$ & $14.8(0.6)$ & $2.0(1.7)^{\dagger \S}$ & $6.2(4.8)^{\dagger}$ \\
\hline Sentence comprehension $(\max =7)$ & $\mathrm{F}(2,20)=5.9^{*}$ & $7.0(0.0)$ & $3.8(2.8)^{\dagger}$ & $5.2(2.7)$ \\
\hline $\begin{array}{l}\text { Modified Rey-Osterrieth Copy ( } \max = \\
\text { 17) }\end{array}$ & $F(2,21)=3.3$ & $15.0(1.2)$ & $16.8(0.4)$ & $13.5(4.2)^{\frac{7}{t}}$ \\
\hline Calculations $(\max =5)$ & $\mathrm{F}(2,21)=0.9$ & $4.5(1.2)$ & $4.5(0.8)$ & $3.7(1.8)$ \\
\hline
\end{tabular}

Values are expressed as mean (SD).

$*$

$p<0.01$ across all groups.

${ }_{p}^{+}<0.05$ vs controls.

$\stackrel{\ddagger}{p}<0.05$ vs LTLV.

$\S_{p}<0.05$ vs RTLV.

LTLV = left temporal lobe variant; RTLV = right temporal lobe variant; ANOVA = analysis of variance; MMSE = Mini-Mental State Examination; CVLT-MS = California Verbal Learning Test-Mental Status version; BNT = Boston Naming Test. 
Table 2

First semantic and behavioral symptoms in temporal variant frontotemporal dementia

\begin{tabular}{|c|c|c|}
\hline \multirow[b]{2}{*}{ Patient } & \multicolumn{2}{|c|}{ First symptom type } \\
\hline & Semantic & Behavioral \\
\hline \multicolumn{3}{|l|}{ LTLV } \\
\hline 1 & Anomia for people, places, objects $*$ & (Disinhibition, compulsions) \\
\hline 2 & Anomia for people, repetitive speech ${ }^{*}$ & Irritability $^{*}{ }^{*}$ \\
\hline 3 & Anomia for people, places, objects ${ }^{*}$ & Insomnia, emotional withdrawal \\
\hline 4 & Word-finding difficulties ${ }^{*}$ & (Weight gain) \\
\hline 5 & Word-finding difficulties, anomia for objects & Hostility/irritability toward husband ${ }^{*}$ \\
\hline 6 & Word-finding difficulties ${ }^{*}$ & $\begin{array}{l}\text { Depression, irritability, suspiciousness, stubbornness, } \\
\text { insensitivity }\end{array}$ \\
\hline \multicolumn{3}{|r|}{ 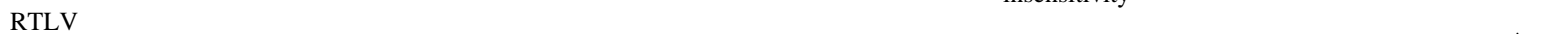 } \\
\hline 1 & Impaired reading/word comprehension & Depression, poor appetite, weight loss, insomnia ${ }^{*}$ \\
\hline 2 & Anomia for people, places, objects ${ }^{*}$ & Apathy \\
\hline 3 & Anomia for people & Emotional withdrawal $^{*}$ \\
\hline 4 & Anomia for people & $\begin{array}{l}\text { Apathy, loss of sexual interest, increased alcohol } \\
\text { intake }\end{array}$ \\
\hline 5 & Anomia, word comprehension ${ }^{*}$ & Irritability $^{*}$ \\
\hline 6 & Impaired word comprehension & Emotional blunting ${ }^{*}$ \\
\hline
\end{tabular}

$\mathrm{LTLV}=$ left temporal lobe variant; $\mathrm{RTLV}=$ right temporal lobe variant. 
Table 3

The nature of compulsions in temporal variant frontotemporal dementia

\begin{tabular}{lll}
\hline & & Compulsion type \\
\cline { 2 - 3 } Patient & Visual: objects as targets & Verbal: words and symbols as targets \\
\hline LTLV & Recycling metal and plastic, stomping ants, weeding, & Solitaire \\
1 & gathering sticks in yard, collecting coins & \\
2 & Dish washing & \\
4 & Gardening, collecting coins & Cataloguing daily event \\
5 & Cleaning & \\
6 & Sharing family photographs & Concern over zip codes, Scrabble, solitaire, writing \\
RTLV & & letters, notes to doctors \\
1 & & Solitaire \\
2 & Skipping cracks in pavement & Decoding word "jumbles" \\
3 & Gathering coins, brightly colored objects & Writing notes, names, and phone numbers \\
4 & & Solitaire, blackjack \\
5 & & Solitaire, Bible quotations \\
\hline
\end{tabular}

LTLV = left temporal lobe variant; RTLV = right temporal lobe variant. 